\title{
React, reset and restore: Adaptation of a large inflammatory bowel disease service during COVID-19 pandemic
}

\author{
Authors: Haidee A Gonzalez, ${ }^{\mathrm{A}}$ Sally Myers, ${ }^{\mathrm{B}}$ Emma Whitehead, ${ }^{\mathrm{C}}$ Alisson Pattinson, ${ }^{\mathrm{C}}$ Katie Stamp, ${ }^{\mathrm{C}}$ \\ Jack Turnbull, ${ }^{C}$ Rebecca Fory, ${ }^{C}$ Bethia Featherstone, ${ }^{C}$ Amy Wilkinson, ${ }^{D}$ Jessica Lisle, ${ }^{\mathrm{E}}$ Greg Haire, ${ }^{\mathrm{F}}$ \\ Eileen Henderson ${ }^{G}$ and Shaji Sebastian ${ }^{H}$
}

\section{Introduction}

Healthcare organisations have had to make adaptations to reduce the impact of the Coronavirus 2019 (COVID-19) pandemic. This has necessitated urgent reconfiguration within inflammatory bowel disease (IBD) services to ensure safety of patients and staff and seamless continuity of care provision.

Aim

To describe the adaptations made by a large inflammatory bowel disease service, caring for over 3,500 IBD patients, in response to the COVID-19 pandemic.

Methods

A diary record of responses to the pandemic were logged, and meeting minutes were reviewed. Data were recorded from IBD advice lines, multidisciplinary team (MDT) meeting minutes, infusion unit attendances, and electronic referral systems for the 8-week period from 9 March 2020 until 2 May 2020. Descriptive analysis was performed.

Results

The IBD service at Hull University Teaching Hospitals NHS Trust (IBD Hull) instituted rapid structural and functional changes to the service. Outpatient services were suspended and substituted by virtual consultations, and inpatient services were reduced and moved to ambulatory care where possible.

The delivery of biologic and immunomodulatory therapies was significantly modified to ensure patient and staff safety. There was a substantial increase in IBD advice line calls.

\section{Conclusion}

The rapidly evolving COVID-19 pandemic required a prompt response, regular reassessment and planning, and continues to do so. We share our experience in of the successful adaptations made to our IBD service.

Authors: ${ }^{A}$ advanced IBD fellow, Hull University Teaching Hospitals NHS Trust, Hull, UK; BIBD research nurse, Hull University Teaching Hospitals NHS Trust, Hull, UK; ' CIBD nurse specialist, Hull University Teaching Hospitals NHS Trust, Hull, UK; DIBD administrator, Hull University Teaching Hospitals NHS Trust, Hull, UK; EIBD clinical trial manager Hull University Teaching Hospitals NHS Trust, Hull, UK; Fservice manager, Hull University Teaching Hospitals NHS Trust, Hull, UK; ' ${ }^{\circ}$ service director, Hull University Teaching Hospitals NHS Trust, Hull, UK; ${ }^{H}$ IBD service lead clinician, Hull University Teaching Hospitals NHS Trust, Hull, UK
KEYWORDS: IBD, COVID-19, adaptations

DOI: $10.7861 /$ clinmed.2020-0369

\section{Introduction}

The ongoing coronavirus 2019 (COVID-19) pandemic is placing unprecedented levels of strain on healthcare systems, which are being faced with significant resourcing challenges. Healthcare organisations have had to make rapid adaptations to the delivery of care to meet these challenges.

For patients with inflammatory bowel diseases (IBD), the current pandemic situation has required reconsideration of how best to deliver diagnosis and medical and surgical treatments. Currently there is no evidence that IBD patients are at higher risk of acquisition of SARS-CoV-2. ${ }^{1-3}$ Emerging data have suggested that the therapies used in IBD may not increase the risk of SARS-CoV-2 infection, nor worsen outcomes. ${ }^{4.5}$ In the UK, the IBD COVID-19 Working Group produced guidelines on the management of IBD during the pandemic and produced a risk grid to enable patients and clinicians to understand each individual's risks. ${ }^{6}$ The Department of Health $(\mathrm{DoH})$ recommended that all high-risk patients were to be shielded for 12 weeks. Nevertheless, there remained understandable concerns among patients and their clinical teams regarding the impact of IBD treatments on the risk of severe COVID-19 disease, particularly for those receiving immunosuppressive or biologic therapies. ${ }^{7}$ As focus and resources were diverted towards the frontline care of COVID-19 patients, the maintenance of safe, high-quality care of IBD patients could prove challenging. ${ }^{8}$

The IBD unit in Hull (IBD Hull) is a dedicated multidisciplinary service with full integration of clinical care and research and covers a primary referral population of 625,000 inhabitants (Fig 1). There are approximately 3,600 IBD patients who are currently under active follow-up with the service out of the 4,220 patients in the pathology database. The clinical care and research functions are fully integrated within the team. The service model has been highly commended by British Society of Gastroenterology. ${ }^{9}$

In this paper, we describe the proactive steps implemented by our IBD service since early March 2020 in response to the COVID-19 pandemic, and provide a viewpoint on how operational changes in IBD management can ensure safe and effective care delivery to IBD patients during the pandemic, without compromising the quality of care, and we report the patients' perceptions of these changes. 


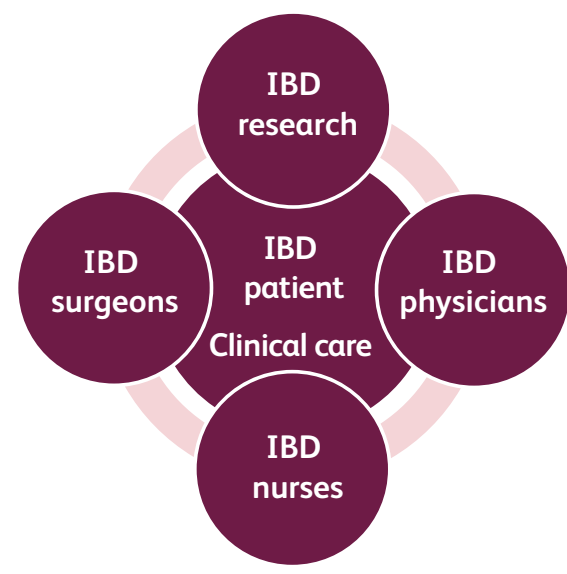

Fig 1. The integrated inflammatory bowel disease (IBD) service.

\section{Methods}

We maintained diary record of the actions and minutes of the IBD unit meetings in relation to the response to the CVOID-19 pandemic. The unit activity data was collected for the 8-week period from 9 March 2020 to 2 May 2020, and, for comparison, we reviewed the corresponding data for the period 4 March 2019 to 22 April 2019. We collected records of patient contact to the IBD advice line and the reasons for the contact. In addition, patients were advised to contact the advice line if they developed symptoms of COVID-19, or they needed to self-isolate following contact with other individuals with suspected or confirmed COVID-19. Data on referrals to the IBD service were collected through the electronic referral system. The immunomodulatory infusion unit database recorded patient attendance to the unit. Staff rota and attendance sheets were reviewed for any absences. We also surveyed 78 consecutive patients attending the services with a patient experience measure questionnaire.

\section{Adaptations to the service}

\section{Planning}

The operational plan for adaptation of the service was discussed and agreed at a virtual meeting carried out using the Zoom videoconferencing platform on 9 March 2020. The basic principles underpinning the plan were safety of patients and staff, avoidance of disruption of care to IBD patients, and ensuring compliance to national guidelines on social distancing and risk stratification. The team was able to gain insights on the key challenges and potential solutions to implementation from international collaborators in Italy, China, and Spain. In the initial 2 weeks of the operational phase, commencing on 20 March 2020, daily Zoom meetings were conducted to identify challenges, review the safety of processes, provide mutual support and share updates, and for educational purposes. After this period, the frequency was reduced to weekly virtual meetings.

The full reconfiguration of the service was implemented by 20 March 2020 to provide the service functions, incorporating inpatient care, outpatient care, infusion service, IBD advice line services, diagnostics, surgery and research (Fig 2).

\section{Staffing}

Temporary suspension of locally sponsored multicentre IBD trials, unrelated to COVID, allowed additional pooling of the research administrative staff to the adaptation team. We assigned two IBD physicians to manage IBD inpatient care and virtual outpatient clinics. Four nurses were responsible for the running of the relocated infusion unit, which also provided urgent clinical assessments and blood tests. One specialist nurse was available to manage the IBD advice line daily, and the data collected from this were collated and communicated to the lead clinician daily by the IBD administrator. At any given time two nursing staff members were available as 'floating' to cover of any absences and sudden surge in activity. Existing annual leave commitments were honoured. During the 2 -month period since initiation of the adaptation model, only 2 days of staff sickness have been reported. No staff have, so far, developed symptoms or tested positive for SARS-CoV-2.

\section{Outpatients}

In line with the local planning, scheduled face-to-face consultations for both new and follow-up patients were cancelled. In the early stage of the pandemic (from 1 March 2020 to 20 March 2020), all the clinics were running to near full capacity by telephone consultation, but this was substantially curtailed to less than

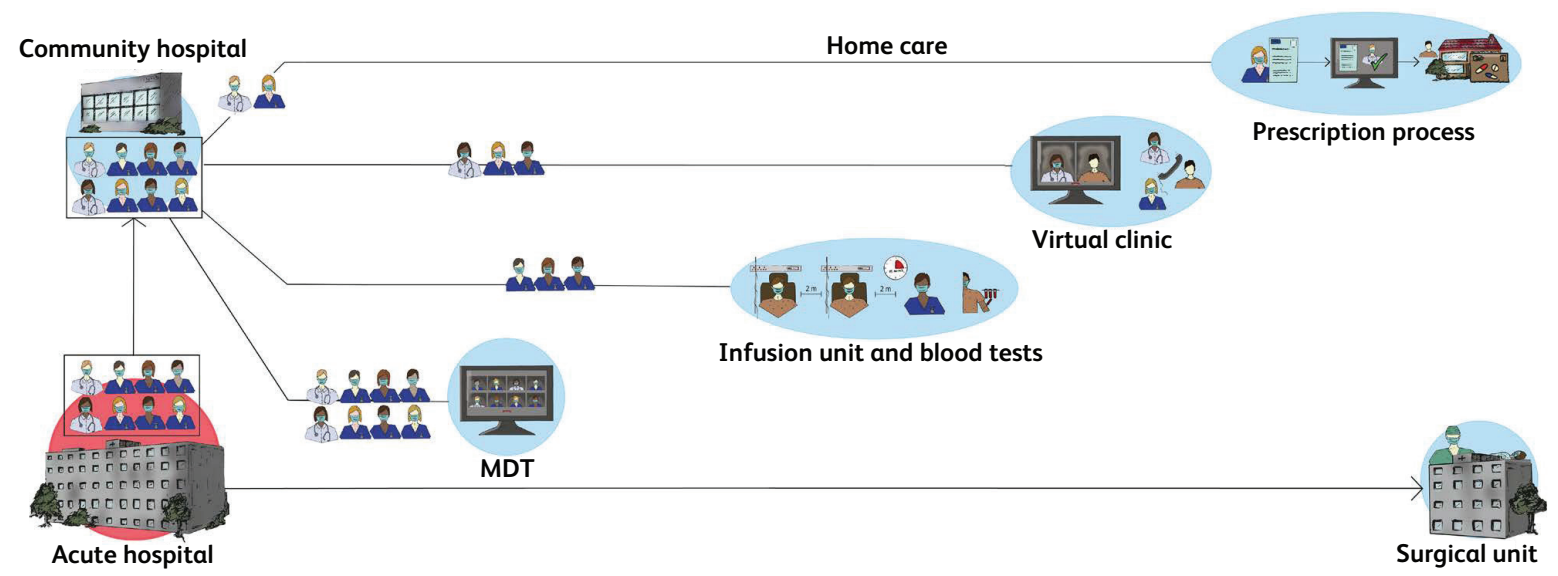

Fig 2. Structural reconfiguration of IBD service. 
$25 \%$ of the normal capacity by 1 April 2020 . All patients received communication by letter, which included a contact e-mail and telephone number for the service so that they could discuss any concerns regarding the cancellations of their appointments. Of the 18 new referrals who had virtual consultations for suspected IBD, 11 patients had calprotectin performed in primary care, and for the remaining seven, calprotectin was arranged by the service. 13 of these patients had diagnostic imaging (three CT scan, four MR enterography) and/or endoscopic evaluation (five sigmoidoscopy, one ileocolonoscopy) arranged. Patients undergoing endoscopic evaluation had nasopharyngeal swabs taken 3 days before their endoscopy. In addition, four virtual consultations for tertiary referral were conducted and treatment modification were made in three patients. Due to the limited availability of endoscopic services, many patients with a prior diagnosis of IBD and symptoms of flare were treated based on the basis of physician global assessment (PGA), faecal calprotectin and inflammatory markers.

Of those who had experienced cancellation of face-to-face follow-up appointments and offered telephone consultations ( $n=46), 92 \%$ rated the experience as 'good' to 'excellent'.

\section{Inpatient care}

Inpatient care was carried out by a general gastroenterology consultant with daily virtual input from the IBD clinician. The IBD nurse support for admitted patients was suspended. Strict isolation policies were implemented for inpatients, with barrier nursing limiting contact with healthcare staff. Six patients were admitted - five with ulcerative colitis (UC) and one with Crohn's disease (CD) - and one patient required colectomy. Five of the six admitted patients were tested for SARS-CoV-2, and none tested positive. Four patients were initiated on biologics during admission, and one patient with small bowel CD was managed with exclusive enteral nutrition and antibiotics. In addition, five additional patients were managed through the ambulatory care acute severe UC (ASUC) pathway, with daily IV methylprednisolone and prophylactic low molecular weight heparin administered at the relocated infusion unit, with daily clinical review and blood monitoring. Four of these patients required rescue therapy with infliximab.

\section{Multidisciplinary team meetings}

Our weekly IBD MDTs were changed to an entirely virtual format. During the 8-week period, 57 patients were discussed at the MDTs (Fig 3). 40 (70.2\%) of these patients were considered suitable to be initiated on biologics, or changed their biologics, and five patients were considered for surgery (Fig 4). The average attendance at MDT was seven, comprising IBD physicians, IBD nurses, an IBD fellow, a colorectal surgeon, a pathologist, and a radiologist. The median time from MDT discussion to the commencement of biologics was 8 days (range 2-15).

\section{Immunomodulatory Infusion Centre (IMIC)}

In order to provide essential intravenous and subcutaneous IBD treatments, away from the acute hospital setting, we relocated our infusion suite to a community facility 12 miles away from the acute unit, which also has provision for phlebotomy services and plain X-rays. The new facility had three treatment rooms and one assessment room, with maximum of two patients per room and

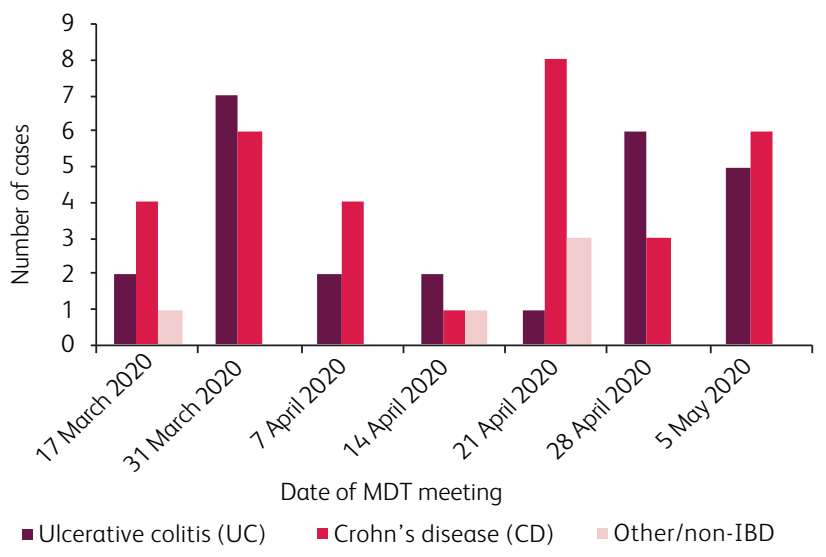

Fig 3. MDT cases by type of disease.

minimum distance of 2 metres between chairs. The appointments were staggered to allow a maximum of only four patients in the facility at any given time. Where required, appointments were rescheduled to prevent overcrowding in the waiting area and to maintain social distancing measures.

All patients attending the unit for treatment had a telephone pre-screen, based on a structured questionnaire, a minimum of 24 hours prior to their scheduled attendance. Patients with symptoms of COVID-19, or history of contact with confirmed or suspected COVID-19 patients, were asked to postpone the visit until they were 7 days without any suggestive symptoms. Access to the facility was restricted to patients only, with relatives and caregivers being restricted entry. Patients were asked to bring their own mask or were provided with a surgical mask on arrival. All patients had their temperature checked and screening questions repeated before proceeding to the infusion area. Safety blood tests and research blood tests, where applicable, were taken at the time of attendance. Personal protective equipment (PPE), as per WHO guidelines, was worn by all members of the IBD team to prevent possible nosocomial infection. The infusion time for infliximab was shortened to the minimum (30 minutes for established patients)

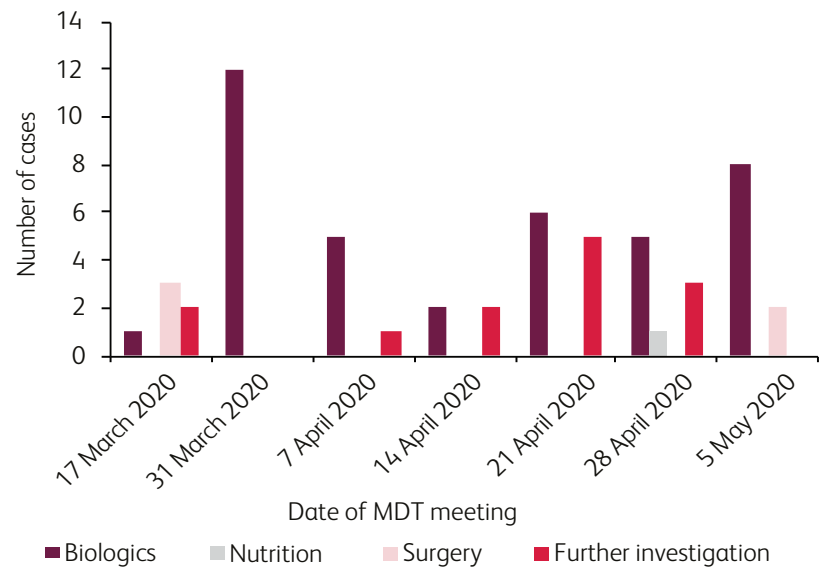

Fig 4. MDT cases by outcome. 


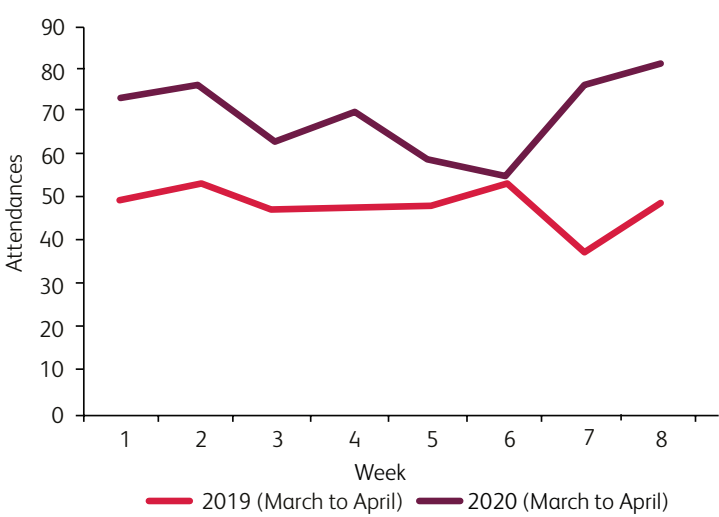

Fig 5. IBD immunomodulatory infusion unit attendances.

where possible. Patients were provided with a dedicated number to contact should they develop any COVID-19 symptoms following their visit to the unit, or if experiencing any delayed adverse events.

Five patients were managed by the ambulatory ASUC pathway using once daily-methyl prednisolone, thromboprophylaxis and daily bloods, abdominal X-rays when indicated and daily clinical review. Four of the five required rescue therapy with infliximab and none required colectomy.

In this 8-week period from 9 March to 2 May 2020, 553 patients attended the relocated immunomodulatory infusion unit, compared to 382 patients during the corresponding period in 2019 (Fig 5). 27 new patients commenced biologics in the study period in comparison to 13 patients during the corresponding period in 2019 (Fig 6). 11 patients had their infusions postponed due to suspected contact with a family member with symptoms suggestive of coronavirus, but no patients who attended the unit have, so far (as of 20 May 2020), tested positive for SARS-CoV-2. Only one patient declined to continue biologics. No new starts for thiopurines or methotrexate were carried out during this period.

The change to the infusion unit has been perceived by our patients as highly positive, with the majority of respondents rating the service as good to excellent for safety (97\%), comfort (92\%) and reduced anxiety (100\%).

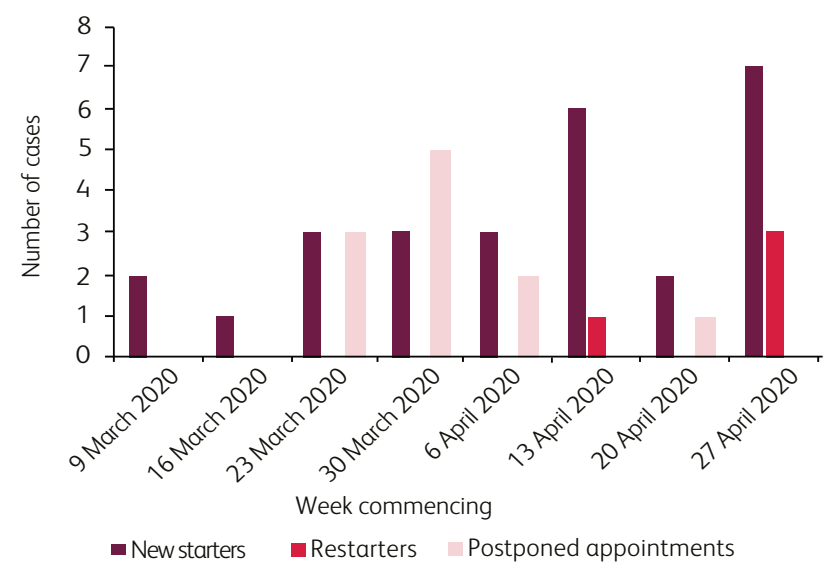

Fig 6. Biologic treatments in immunomodulatory infusion unit. W/C = week commencing.

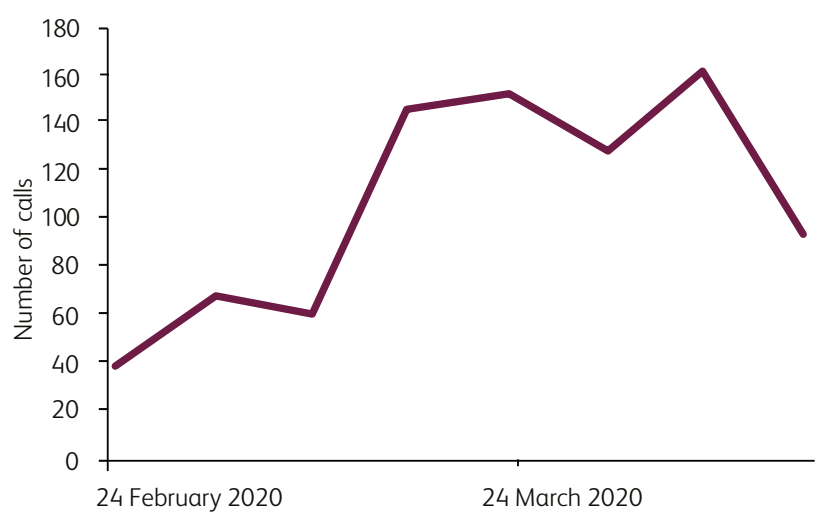

Fig 7. Number of helpline calls.

\section{IBD advice line}

The advice line was reconfigured to provide contact via email and telephone, with additional automated messages incorporating general advice related to COVID-19. A minimum of one IBD nurse managed the helpline along with an administrator to support the e-advice line, and contact was made with patients within 24 hours in almost all cases.

There was a fourfold increase in advice line contacts during the study period, increasing from 58 calls in February 2020 to 238 and 233 calls in March and April 2020, respectively (Fig 7). 59\% of the calls during the study period were for COVID-19 related queries, $13.4 \%$ were related to IBD flares, and $27.8 \%$ were related to advice about medications including home supply of biologics and supply issues of thiopurines (Fig 8).

\section{Risk stratification}

In order to identify patients who required shielding, all members of the team were allocated patients to screen for comorbidity, current disease activity, current steroid use, and admission within the previous 6 weeks. All patients were sent a letter, encouraging them to use the risk identification tool from Crohn's and Colitis UK, and advised to contact the helpline with their risk identification (which was then verified by the team). Shielding letters were sent to patients on 24 March 2020, and a further letter with the BSG

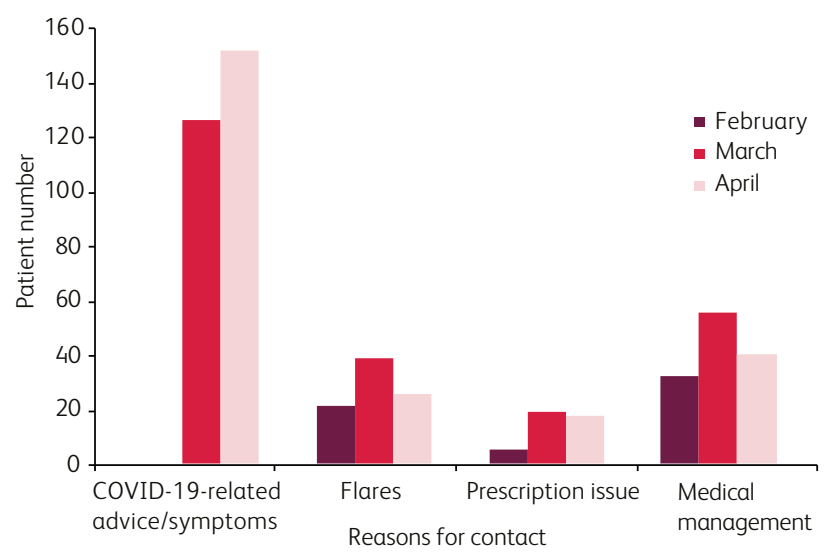

Fig 8. Reason for use of IBD advice line. 
risk grid with self-identification tool send to all patients by the first week of April. Changes in patient risk status following admission, advice line status, or through communication from the BSG risk grid tool were recorded on a weekly basis on a central database.

Overall, there was a high rate of satisfaction from the patients regarding the information provided. $95 \%$ stated they had received understandable information about their level of risk; $92 \%$ felt the IBD service had provided them with enough information and guidance concerning the impact of COVID19 on their IBD and treatment, and $91 \%$ rated their understanding with regard to social distancing and self-isolation, and the information with which they had been provided, as good to excellent.

\section{Research}

In line with recommendations from NIHR and the hospital guidelines, all non-COVID recruitment to clinical trials was suspended. Patients on clinical trial medications were continued on the trial medications in discussion with the sponsors. Monitoring activity was conducted virtually. Four multicentre studies sponsored by the service have been suspended. The service is leading, or participating in, seven COVID-19 research projects including PREPARE IBD (www.prepareibd.org), and participating in the international registry of IBD patients with COVID-19, the SECURE IBD registry (www.covidibd.org). The service has led or contributed to four peer-reviewed publications $s^{6,10-12}$ so far.

\section{Patient education}

In line with DoH guidelines, all IBD patients in our cohort have been contacted and provided with the information they require in relation to social distancing, shielding and their current management. The disease specific information was posted on the Trust website, and social media posts via Twitter and Facebook were utilised. All our patients have had access to the IBD Telephone helpline and the IBD patient email service enabling them to communicate effectively with the team and source the correct information. Charity partners such as Crohn's and Colitis UK have also published information. ${ }^{13}$

\section{Post-pandemic recovery plans}

In discussion with all stakeholders, and including patients, we have developed the following 10-point post-pandemic recovery plan.

> Virtual consultation (telephone and video consultation) to be upscaled to $75 \%$ of the outpatient appointments.

> Uptake of the ambulatory care pathway for admitted patients with active IBD to be increased, aiming to reach $30 \%$ of admissions.

> Staged endoscopic assessment to be based on strict criteria, on dedicated IBD specialist endoscopy lists starting with new diagnosis, flare assessments and clinical trial assessments.

$>$ IBD advice line access to be reconfigured to provide increased access

$>$ IBD-consultant-led new patient triage for patients with suspected IBD to be carried out.

> The immunomodulatory infusion unit to be allocated a more expansive footprint, in order to maintain social distancing.

> Uptake of self-administered therapies and self-management options for patients to be increased.

> Patient-held applications to support virtual and digital IBD service to be developed.
> Research processes and protocols to be modified to ensure restoration and enhancement of research activity.

> Support structures for patients and staff, including psychological support, to be developed.

\section{Discussion}

COVID-19 is changing the face of healthcare delivery, acting as a catalyst for innovation, and, now that it is unlikely that we will put the 'genie back in the bottle', the changes represent a remarkable reconfiguration of healthcare delivery which may prove to be one of the most radical long-term changes to the way health services are delivered following COVID-19. IBD services across the UK, and indeed across the world, have needed to adapt to meet the challenges posed by the COVID-19 pandemic. ${ }^{14}$ In this paper, we report our experience in the first 60 days of service adaptation in a large-volume IBD centre.

The team was able to learn from international collaborators about the pattern of the pandemic in China, Italy, and Spain..$^{14-16}$ Armed with that information, we were able to able to implement rapid adaptations to the service. However, the lack of data on the risks to IBD patients from SARS-CoV-2, in particular on possible mitigation strategies to protect patients on immunomodulators and biologics, was a barrier in earlier implementation of changes. Our participation in developing the UK IBD risk grid ${ }^{6}$ and consensus statements from IOIBD ${ }^{17}$ provided much-needed clarity. Clear and frequent communication with the whole team, using frequent virtual meetings and group messaging platforms, ensured that the key messages were acted upon promptly early in the pandemic before the region reported a peak in its cases.

The move of the infusion unit to a safer environment away from the acute care setting, coupled with strict infection control and public health measures for staff and patients alike, ensured that treatments were given without interruption in a safer environment. This, along with clear, repeated and consistent messaging also potentially reduced patient anxiety and maintained treatment adherence. We conducted a patient-reported experience measure survey which indicated high levels of satisfaction to the changes (results submitted for separate publication).

As reported in other IBD centres ${ }^{7}$ and noted in the recent UK survey, ${ }^{11}$ there was an exponential increase in the volume of contact with IBD advice lines at the beginning of the pandemic. Consistent messaging through different modes of communication that we implemented have resulted in this returning to regular activity figures. The advice line continues to be a major channel for patient interaction with the team, including early identification and management of flares and early reports of symptoms requiring isolation.

In line with national guidance, endoscopy and surgical services have been largely put on hold. The clinical impact of this on patients is yet to be quantified. There is an emerging consensus on endoscopy provision for IBD patients during the COVID-19 pandemic and for the post-pandemic period. ${ }^{18}$

The post-COVID world is likely to be remembered as the time when other_medical interactions, including routine outpatient care, shifted to digital modalities as the default rather than the exception. We used telephone clinics, but the development of video consultations will be a welcome addition. While telehealth modalities have previously been used in IBD, ${ }^{19}$ the pandemic will accelerate their adoption.

Our report may not be applicable to all IBD services. The numbers of IBD specialist staff, in particular IBD specialist nurses, vary widely in 
hospitals around the UK.11 The integrated model of our service, which may not be available in some centres, allowed us to pool resources and reassign new roles to staff already familiar with the clinical practice environment. Several healthcare professionals have been redeployed to frontline COVID-19 duties which may negatively impact on provision of specialist services. We were able to maintain the full staff complement of the IBD service without redeployment. We did not have significant staff sickness or absence due to COVID-19. The physical and psychological impact of the pandemic on healthcare staff should be a major consideration in current and future planning.

Some of the service changes we implemented may be applicable to many IBD units in the event of a second peak of the current pandemic or a new pandemic. The key was early proactive adoption of changes to meet specific challenges in the functions of the service impacted by the pandemic and making adaptations for the safe delivery of treatments.

\section{Conclusions}

The COVID-19 pandemic will continue to spread worldwide, imposing increasing pressures and burden on healthcare providers. To face the challenges which lie ahead, IBD teams need to rapidly adapt to the ever-evolving situation through collective partnership with their patients and the wider multidisciplinary teams. Communication between the service user and the care provider is fundamental to care delivery and safe IBD management. As a result of the measures put in place, as highlighted above, we continue to effectively maintain and deliver a high quality of care to our IBD cohort. Further data on the incidence and prognosis of COVID-19 in patients with IBD is needed to guide our practice and develop our understanding of the overall impact of COVID-19 and IBD.

\section{Acknowledgements}

The authors acknowledge the patients and their care givers for their understanding and co-operation during this challenging period. We also want to thank Ms Molly Talbott for the infographics in Fig 2.

\section{Conflicts of interest}

Haidee A Gonzalez has received lecture fees from Falk Pharma, Pfizer, Takeda and Nestle. Sally Myers and Emma Whitehead have received speaker fees from Janssen. Shaji Sebastian holds research grants from Biogen, Takeda, AbbVie, Tillotts Pharma, Ferring and Biohit; served on the advisory boards of Takeda, AbbVie, Merck, Ferring, Pharmacocosmos, Warner Chilcott, Janssen, Falk Pharma, Biohit, TriGenix, Celgene and Tillots Pharma; and has received speaker fees from AbbVie, Biogen, AbbVie, Janssen, Merck, Warner Chilcott and Falk Pharma.

\section{References}

1 Monteleone G, Ardizzone S. Are patients with inflammatory bowel disease at increased risk for Covid-19 infection? J Crohns Colitis 2020, in press (doi: 10.1093/ecco-jcc/jjaa061).

2 Norsa L, Indriolo A, Sansotta $\mathrm{N}$ et al. Uneventful course in IBD patients during SARS-CoV-2 outbreak in northern Italy. Gastroenterology 2020, in press (doi: 10.1053/j.gastro.2020. 03.062).
3 Taxonera C, Sgastagoitia I, Alba C et al. 2019 novel coronavirus disease (COVID-19) in patients with inflammatory bowel diseases. Aliment Pharmacol Ther 2020; 52:276-83.

4 Bezzio C, Saibeni S, Variola A et al. Inflammatory bowel disease outcomes of COVID-19 in 79 patients with IBD in Italy: an IG-IBD study. Gut 2020;69:1213-7.

5 Rodríguez-Lago I, Ramírez de la Piscina P, Elorza A et al. Characteristics, and prognosis of patients with inflammatory bowel disease during the SARS-CoV-2 pandemic in the Basque Country (Spain). Gastroenterology 2020, in press (doi: 10.1053/j. gastro.2020.04.043).

6 Kennedy NA, Jones GR, Lamb CA et al. British Society of Gastroenterology guidance for management of inflammatory bowel disease during the COVID-19 pandemic. Gut 2020;69:984-90.

7 D`Amico F, Danese S, Peyrin-Biroulet L, on behalf of the ECCO COVID19 taskforce. Inflammatory bowel disease management during the COVID19- outbreak: a survey from the European Crohn's and Colitis Organization (ECCO). Gastroenterology 2020, in press (doi: 10.1053/j.gastro.2020.04.059).

8 Allocca M, Fiorino G, Furfaro F et al. Maintaining the quality standards of care for inflammatory bowel disease patients during the COVID-19 pandemic. Clin Gastroenterol Hepatol 2020;18:1882-3.

9 Sebastian S, Giaffer M, Myers S et al. Service success stories: Integrated Clinical and Research IBD Unit. www.bsg.org.uk/clinicalresource/integrated-clinical-and-research-ibd-unit/.

10 Sebastian S, Gonzalez HA, Peyrin-Biroulet L. Safety of drugs during previous and current coronavirus pandemics: Lessons for IBD. J Crohns Colitis 2020, in press (doi: 10.1093/ecco-jcc/jjaa120).

11 Kennedy NA, Hansen R, Younge L, et al. Organisational changes and challenges for inflammatory bowel disease services in the UK during the COVID-19 pandemic. Frontline Gastroenterol 2020, in press (doi: 10.1136/flgastro-2020-101520).

12 Maurice JB, Siau K, Sebastian S et al. Green endoscopy: a call for sustainability in the midst of COVID-19. Lancet Gastroenterol Hepatol 2020;5:636-8.

13 Crohn's on Colitis UK. Coronavirus (COVID-19): FAQs for people with Crohn's and Colitis. www.crohnsandcolitis.org.uk/news/ coronavirus-covid-19-advice.

14 The Lancet. COVID-19: learning from experience. Lancet 2020;395:1011.

15 Danese S, Ran ZH, Repici A et al. Gastroenterology department operational reorganisation at the time of Covid-19 outbreak: an Italian and Chinese experience. Gut 2020;69:981-3.

16 Danese S, Cecconi M, Spinelli A. (2020, 3 25). Management of IBD during the COVID-19 outbreak: resetting clinical priorities. Nat Rev Gastroenterol Hepatol 2020; 17:253-5.

17 Rubin D, Abreu M, Rai V, Siegel C. Management of patients with Crohn's disease and ulcerative colitis during the COVID-19 pandemic: results of an international meeting. Gastroenterology 2020, in press (doi: 10.1053/j.gastro.2020.04.002).

18 Iacucci M, Cannatelli R, Labarile $\mathrm{N}$ et al. Endoscopy in inflammam tory bowel diseases during the COVID-19 pandemic and postpandemic period. Lancet Gastroenterol Hepatol 2020;5:598-606.

19 Cross RK, Langenberg P, Regueiro $\mathrm{M}$ et al. A randomized controlled trial of TELEmedicine for patients with Inflammatory Bowel Disease (TELE-IBD). Am J Gastroenterol 2019;114:472-82.

Address for correspondence: Prof Shaji Sebastian, IBD Unit, Department of Gastroenterology, Hull Royal Infirmary, Hull University Teaching Hospitals NHS Trust, Anlaby Road, Hull HU3 2JZ, UK.

Email: shaji.sebastian@hey.nhs.uk 